\title{
Deduction of the rates of radial diffusion of protons from the structure of the Earth's radiation belts
}

\author{
Alexander S. Kovtyukh \\ Skobeltsyn Institute of Nuclear Physics, Moscow State University, 119899, Moscow, Russia \\ Correspondence to: Alexander S. Kovtyukh (kovtyukhas@mail.ru)
}

Received: 12 May 2016 - Revised: 31 October 2016 - Accepted: 1 November 2016 - Published: 23 November 2016

\begin{abstract}
From the data on the fluxes and energy spectra of protons with an equatorial pitch angle of $\alpha_{0} \approx 90^{\circ}$ during quiet and slightly disturbed $(\mathrm{Kp} \leq 2)$ periods, I directly calculated the value $D_{L L}$, which is a measure of the rate of radial transport (diffusion) of trapped particles. This is done by successively solving the systems (chains) of integrodifferential equations which describe the balance of radial transport/acceleration and ionization losses of low-energy protons of the stationary belt. This was done for the first time. For these calculations, I used data of International SunEarth Explorer 1 (ISEE-1) for protons with an energy of 24 to $2081 \mathrm{keV}$ at $L=2-10$ and data of Explorer-45 for protons with an energy of 78.6 to $872 \mathrm{keV}$ at $L=2-5$. Ionization losses of protons (Coulomb losses and charge exchange) were calculated on the basis of modern models of the plasmasphere and the exosphere. It is shown that for protons with $\mu$ from $\sim 0.7$ to $\sim 7 \mathrm{keVnT}^{-1}$ at $L \approx 4.5-10$, the functions of $D_{L L}$ can be approximated by the following equivalent expressions: $D_{L L} \approx 4.9 \times 10^{-14} \mu^{-4.1} L^{8.2}$ or $D_{L L} \approx$ $1.3 \times 10^{5}(E L)^{-4.1}$ or $D_{L L} \approx 1.2 \times 10^{-9} f_{\mathrm{d}}^{-4.1}$, where $f_{\mathrm{d}}$ is the drift frequency of the protons (in $\mathrm{mHz}$ ), $D_{L L}$ is measured in $\mathrm{s}^{-1}, E$ is measured in kiloelectronvolt and $\mu$ is measured in kiloelectronvolt per nanotesla. These results are consistent with the radial diffusion of particles under the action of the electric field fluctuations (pulsations) in the range of Pc6 and contradict the mechanism of the radial diffusion of particles under the action of sudden impulses (SIs) of the magnetic field and also under the action of substorm impulses of the electric field. During magnetic storms $D_{L L}$ increases, and the expressions for $D_{L L}$ obtained here can change completely.
\end{abstract}

Keywords. Magnetospheric physics (energetic particles trapped)

\section{Introduction}

In the first stage (in the 1960s), exploration of Earth's radiation belts was very active and culminated with the construction of a general dynamic picture of these belts and the creation of a classical theory of this natural particle accelerator.

In the 1970s and 1980s measurements of fluxes and energy spectra of the trapped particles were continued. Detailed measurements of pitch-angle distributions of electrons and protons were carried out. The following were studied in detail: the dynamics of the belts during storms, cyclotron instability and precipitation of particles from the belts, dynamics of the ion composition of the belts, the ring current during storms and substorms, and stochastic effects of drift motion of trapped particles. However, in these decades it seemed that all the basic problems of the physics of the Earth's radiation belts were solved, at least for proton belts, and it remained only to clarify some of the details, accurately carrying out mathematical modeling of the belts and constructing a dynamic mathematical and empirical models.

In the early 1990s surprising dynamical effects of electrons and protons with energies of tens of megaelectronvolt were suddenly discovered in the depths of the Earth's radiation belts (Blake et al., 1992), and further studies showed very complex and in many respects uncertain dynamics of the outer belt of relativistic electrons. These discoveries led to a revision of the classical theory, including problems related to the transport and acceleration of particles. Since the basic properties of the mechanisms of this transport and acceleration are universal for all particles of the Earth's radiation belts, such a revision also concerns the ion belts.

According to the classical theory, the Earth's radiation belts are formed by mechanisms of the radial diffusion of particles under the action of fluctuations of electric and magnetic 
fields in the range of the drift periods of trapped particles, i.e., in the range from several minutes to some hours (Tverskoy, 1969; Roederer, 1970; Schulz and Lanzerotti, 1974; Walt, 1994). Only protons with $E>10 \mathrm{MeV}$ and electrons with $E<0.8 \mathrm{MeV}$ at $L<2$ related to the mechanism of cosmic rays albedo neutron decay (CRAND) are an exception.

At the same time, the first $(\mu)$ and the second ( $I$ or $K=I / p$, where $p$ is the momentum of a particle) invariants of the drift motion of particles are conserved, and the third invariant $(\Phi)$ is violated. The first invariant is associated with the gyration of charged particles in a magnetic field, the second invariant is associated with the oscillations of the particles between the mirror points, and the third invariant is associated with the drift of the particles around the Earth in a magnetic trap. The drift shell parameter $L$ is related to the invariant $\Phi$ by a well-known linear expression (Roederer, 1970).

Radial diffusion of trapped particles is determined by their resonant interaction with the fluctuations of electric and magnetic fields on the drift frequencies of these particles. The main parameter of a radial diffusion $\left(D_{L L}\right)$ determines the rates of radial transport of the trapped particles and in the general case, such as the drift frequency, $D_{L L}$ depends on $L, \mu, K$ and the electric charge of the particles. If small relativistic corrections are neglected, the drift frequency of the particles and $D_{L L}$ do not depend on the rest mass of the particles and are applicable to both protons and electrons.

The parameter $D_{L L}$ is determined by the specific mechanisms of diffusion and is changed with the level and pattern of magnetic activity, as well as changes in solar wind parameter and the interplanetary magnetic field (IMF). The value of $D_{L L}$ can be increased by several orders of magnitude during strong magnetic activity (e.g., Tverskoy, 1969; Lanzerotti et al., 1978; Walt, 1994). It also depends on the phase of the solar cycle, the state of the ionosphere, and the spectral density of electromagnetic fluctuations (pulsations) in the range of ultralow frequency (ULF).

The first evaluations of $D_{L L}$ was obtained by groundbased data of low-frequency fluctuations of the magnetic field (Nakada and Mead, 1965; Tverskoy, 1965). These estimates differ from each other by 1 order of magnitude.

The spectra of the fluctuations of magnetic and electric fields in the range of ULF were also obtained from satellites (e.g., Lanzerotti et al., 1978; Holzworth and Mozer, 1979; Lanzerotti and Wolfe, 1980; Ali et al., 2015). The results of these estimates of $D_{L L}$ differ from each other by several orders of magnitude.

In recent years, in connection with the problem of the dynamics of the outer belt of relativistic electrons, this work intensified. On the basis of spectra of pulsations of the magnetic and electric fields in the range of ULF (Pc4-Pc5), values of $D_{L L}$ have been calculated in many recent works (e.g., Tu et al., 2012; Ozeke et al., 2012, 2014; Ali et al., 2015; Liu et al., 2016). For this purpose, data from the Geostationary Operational Environmental Satellite (GOES), Active Magne- tospheric Particle Tracer Explorers (AMPTE), the Combined Release and Radiation Effects Satellite (CRRES), the Time History of Events and Macroscale Interactions during Substorms (THEMIS) mission, Van Allen Probes, etc., for spectra of pulsations are used. The results of these calculations of $D_{L L}$ also differ significantly from each other.

The parameter $D_{L L}$ was also evaluated as a result of a numerical solution of the radial diffusion equation and fitting it to the experimental data on the fluxes and energy spectra of the Earth's radiation belts. This work was done in the same way as for electrons (e.g., Newkirk and Walt, 1968; Lanzerotti et al., 1970; Tomassian et al., 1972; West et al., 1981; Chiu et al., 1990; Brautigam and Albert, 2000; Brautigam et al., 2005; Ma et al., 2016), protons and other ions/nuclei (e.g., Spjeldvik, 1977; Fritz and Spjeldvik, 1981; Jentsch, 1981; Westphalen and Spjeldvik, 1982; Panasyuk, 2004; Alinejad and Armstrong, 2006; Selesnick et al., 2016). The values of $D_{L L}$ obtained by this method differ from each other by 2 and more orders of magnitude.

In overall mathematical modeling of the Earth's radiation belts, as in the project SALAMMBO, $D_{L L}$ is a result of the selection and variation of certain classes of functions in the framework of a set of calculations that takes into account all known factors affecting the belts (e.g., Beutier et al., 1995). The results of such computations depend on many free parameters which vary during the calculations.

I solved the inverse problem: the values of $D_{L L}$ are derived directly from experimental data on the fluxes and spectra of the trapped protons. Methods of mathematical modeling are not used here. There are no free parameters here. In the discussion of the obtained results, I consider only the most reliable conclusions found from experimental data and the most general physical reasons. After deriving $D_{L L}$ from the proton data (Sect. 2), I discuss the obtained results and compare them with data on the fluctuations (pulsations) of electric and magnetic fields in the range of ULF (Sect. 3).

\section{The calculation of $D_{L L}(\mu, L)$ from the structure of the proton belt}

To extract $D_{L L}$ from the data on trapped particles, using the diffusion equation, it is necessary to have complete and reliable values of the rate of loss of these particles depending on $L$ at various fixed values of $\mu$. For the proton belt near the equatorial plane such dependences are presented in Kovtyukh (2016) for quiet periods. These dependences were calculated on the basis of modern models of distributions of cold plasma and atoms in the geomagnetic trap.

These calculations take into account that for the quiet belt the main loss mechanism of protons is the ionization losses. During the quiet periods, proton precipitation and the influence of ion cyclotron and other waves on the lifetimes of protons can be neglected (e.g., Schulz and Lanzerotti, 1974; Lyons and Williams, 1984). 
The values of $D_{L L}(\mu, L)$ for the proton belt are calculated here on the basis of results in Kovtyukh (2016), satellite data and a diffusion equation. For these calculations, I used data of the International Sun-Earth Explorer 1 (ISEE-1) for protons with an energy of 24 to $2081 \mathrm{keV}$ at $L=2-10$ (Williams, 1981; Williams and Frank, 1984) and data of Explorer-45 for protons with an energy of 78.6 to $872 \mathrm{keV}$ at $L=2-5$ (Fritz and Spjeldvik, 1981). These data are verified in different studies and are in good agreement with each other.

Radial diffusion of the particles is described by the Fokker-Planck equation (e.g., Tverskoy, 1964; Roederer 1970; Schulz and Lanzerotti, 1974). Under certain conditions, which are fully implemented for these protons, the equation is reduced to the ordinary diffusion equation (e.g., Tverskoy, 1965; Fälthammar, 1968; Roederer, 1970; Schulz and Lanzerotti, 1974).

The values of $D_{L L}$ are most simply derived from the data obtained near the equatorial plane. Here, I will consider only protons with equatorial pitch angles of $\alpha_{0}$ close to $90^{\circ}$ (particles with the second adiabatic invariant $K \approx 0$ ).

On the basis of numerous experimental results, I believe that in quiet $(\mathrm{Kp}<2)$ periods the belt of protons with $\alpha_{0} \sim 90^{\circ}$ and $E \sim 0.1-1 \mathrm{MeV}$ is almost stationary. I also believe that local sources of these protons are absent at $2<L<10$.

In this case, radial diffusion and losses of the protons are described by the following equation:

$L^{2} \frac{\partial}{\partial L}\left(\frac{D_{L L}}{L^{2}} \frac{\partial f}{\partial L}\right)=-\left(\frac{\partial f}{\partial t}\right)_{\mathrm{cc}}-\left(\frac{\partial f}{\partial t}\right)_{\mathrm{ce}}$,

where $f(\mu, L)$ is the distribution function of protons in the phase space. The functions $f$ and $D_{L L}$ in this equation refer to the particles with given values of $\mu$. Equation (1) shows that for each $L$ shell of the stationary radiation belt, diffusion and losses of protons with given values of $\mu$ are completely balanced.

The first term on the right-hand side of Eq. (1) describes Coulomb losses of protons, and the second term describes the charge exchange of protons with atoms. Coulomb scattering of protons by pitch angles is neglected in Eq. (1) according to Schulz and Lanzerotti (1974).

The proton loss rate depends on the distributions of cold plasma and atoms in the geomagnetic trap. Modern models of these distributions are the most reliable for magnetically quiet periods. During geomagnetic disturbances the distributions change (the distribution of cold plasma changes very much, and the density of atoms varies within $20 \%$ ).

Losses related to ion-cyclotron waves are also added during geomagnetic disturbances.

With the increase in geomagnetic activity, the values of $D_{L L}$ increase, and the magnitude of the effect may depend on $L$.

Thus, in order to finding $D_{L L}(\mu, L)$ for the trapped protons, functions $f(\mu, L)$ of protons and the rates of ionization losses of protons with different $\mu$ on different $L$ must first be calculated with the satellite data obtained near the plane of the geomagnetic equator in the magnetically quiet periods.

I will consider the protons with $\mu$ from 0.2 to $7 \mathrm{keV} \mathrm{nT}^{-1}$ (from 20 to $700 \mathrm{MeV} \mathrm{G}^{-1}$ ) and $L \approx 2.5-10$. These particles are adjacent and in part overlap with less energetic particles, which are usually attributed to the storm ring current (e.g., Williams, 1987). During the quiet periods, the protons considered here are the major contributors to the pressure of the trapped particles in the geomagnetic trap. Therefore, they can be regarded as a quiet ring current (see Kovtyukh, 2001). This belt of protons is called the ring current also in Williams (1981), the results of which are used in my work. However, at $\mu>0.5 \pm 0.2 \mathrm{keVnT}^{-1}$ at $L>3$, a belt of protons (and other trapped ions) in quiet and slightly disturbed periods is maintained in the stationary state mainly due to the radial diffusion of particles from the outer boundary of the trap to the Earth with conservation $\mu$ and $K$ (Kovtyukh, 2001).

\subsection{The calculation of $f(\mu, L)$ for the belt of protons}

For calculations of $f(\mu, L)$, I took the data of Explorer-45 and ISEE-1 for a protons with $\alpha_{0} \approx 90^{\circ}(K \approx 0)$. The data of Explorer-45 are obtained for June 1972, near the maximum (early fall) of the 20th cycle of solar activity; the data of ISEE-1 are obtained for November 1977, at a minimum (at the beginning of the growth) of the 21st cycle.

For nonrelativistic protons with $\alpha_{0}=90^{\circ}(K=0)$

$f(\mu, L)=k \frac{j[L, E(\mu, L)]}{E(\mu, L)}=k f^{*}(\mu, L)$,

where $j[L, E(\mu, L)]$ is the measured fluxes of protons, $E$ is the kinetic energy of protons, and

$\mu\left(\mathrm{keV} \cdot \mathrm{nT}^{-1}\right)=\frac{E}{B_{0}(L)}=3.215 \times 10^{-5} L^{3} E(\mathrm{keV})$,

where $B_{0}(L)$ is the magnetic induction near the equatorial plane. The values of $\mu$ were calculated here for the dipole magnetic field.

A value of the coefficient $k$ depends on dimensions of variables in Eq. (2). For $j$ given as $\left(\mathrm{cm}^{2} \mathrm{~s} \times \operatorname{ster} \times \mathrm{keV}\right)^{-1}, E$ given in kiloelectronvolt and $f$ given as $\mathrm{s}^{3} \mathrm{~cm}^{-6}$, the value of $k=5.447 \times 10^{-31}$. The coefficient $k$ plays no role in our calculations, so I will use $f^{*}(\mu, L)$ instead of $f(\mu, L)$. Equation (1) is invariant under this replacement.

In Kovtyukh (2016) functions $f^{*}(\mu, L)$ of the trapped protons were calculated on the basis of the ISEE-1 data for the quiet period $(\mathrm{Kp} \leq 1)$, from 20:27 UT 24 November 1977 to 01:30 UT 25 November 1977, given in Williams (1981). For completeness, here I used also ISEE-1 data for a weakly disturbed period from 17:52 to 21:05 UT 17 November 1977 (Williams and Frank, 1984). In this period the index Dst has changed from -17 to $-18 \mathrm{nT}$, and the index $\mathrm{Kp}=1_{-}$ $\left(\mathrm{Kp} \leq 2_{+}\right.$for $12 \mathrm{~h}$ and $\mathrm{Kp} \leq 3_{-}$for $24 \mathrm{~h}$ prior to this period 


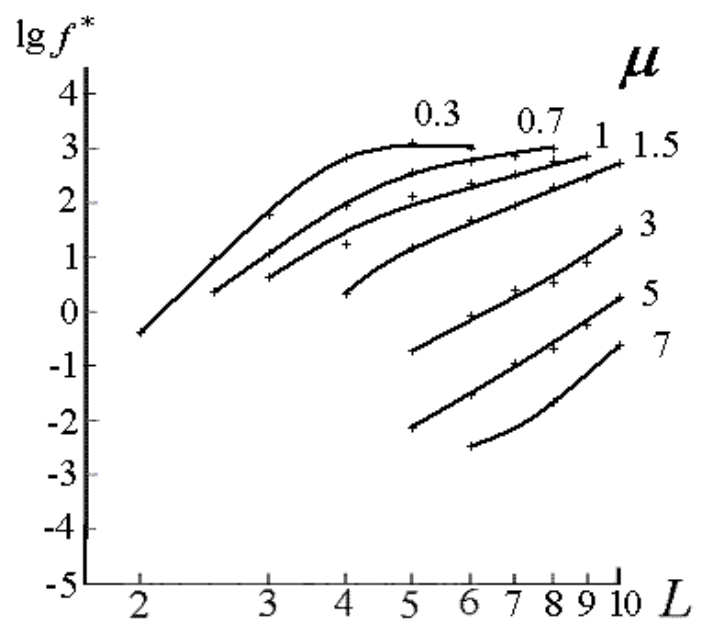

Figure 1. Functions $f^{*}(L)$ for protons with different $\mu$ (from 0.3 to $7 \mathrm{keV} \mathrm{nT}^{-1}$ ) calculated with ISEE-1 data from Williams and Frank (1984) for a slightly disturbed $(\mathrm{Kp} \leq 2)$ period 17 November 1977. Here $\mu$ is given in kiloelectronvolt per nanotesla. The value of $f^{*}\left(\mathrm{~s}^{3} \mathrm{~cm}^{-6}\right)$ is $1.836 \times 10^{30} f$.

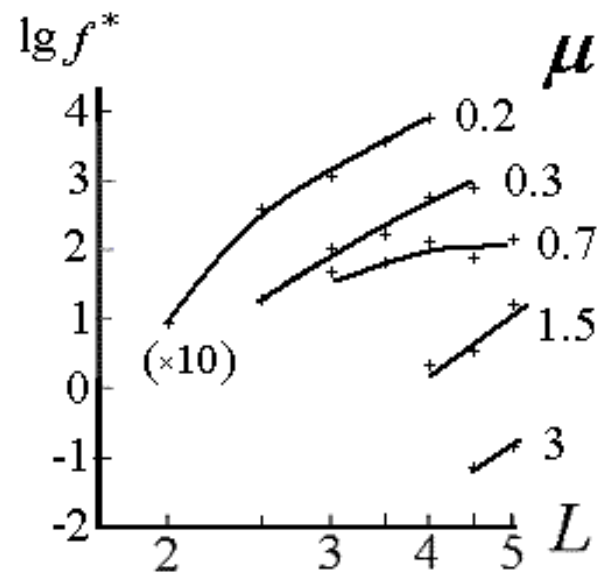

Figure 2. Functions $f^{*}(L)$ for protons with different $\mu$ (from 0.2 to $3 \mathrm{keV} \mathrm{nT}^{-1}$ ) calculated with Explorer-45 data from Fritz and Spjeldvik (1981) for the quiet period of 1-15 June 1972. Here $\mu$ is given in kiloelectronvolt per nanotesla. The value of $f^{*}\left(\mathrm{~s}^{3} \mathrm{~cm}^{-6}\right)$ is $1.836 \times 10^{30} \mathrm{f}$. The curve for $\mu=0.2 \mathrm{keV} \mathrm{nT}^{-1}$ is raised above the other curves by 1 order of magnitude.

UT). The measurements were carried out near the noon sector, in the following eight energy channels: 24-45.5-65.395.5-142-210-333-849-2081 keV.

For calculating the functions $f^{*}(\mu, L)$, it is necessary to have the differential fluxes of particles (see Eq. 2). As a rule, to find these fluxes the count rate of particles in each channel is divided by the geometric factor of the instrument and by the width of the corresponding channel. The values thus obtained refer to the midpoints of the channels, i.e., to the arithmetic mean values of the energy channels, $\bar{E}=\left(E_{1}+E_{2}\right) / 2$, where $E_{1}$ and $E_{2}$ are the lower and upper bounds of this channel. But this is true only for a flat spectrum or for the linear spectrum, i.e., $j(E) \propto E$. Sometimes the energy of the particles in the channel is defined as the geometric mean, $\bar{E}=\sqrt{E_{1} E_{2}}$, but this is true only for the power spectrum $j(E) \propto E^{-2}$. For a more accurate binding of the experimental data to a specific energy of the particles (within each channel of the spectrometer) and calculations of the functions $f^{*}(\mu, L)$, I have developed a special method based on successive approximations to the integrating fluxes within the energy channels of the device. This method is described in detail in Kovtyukh (2016).

Using the ISEE-1 data of Williams and Frank (1984) for the period 17:52-21:05 UT 17 November 1977, I have calculated the $f^{*}(\mu, L)$ for protons with $\mu$ from 0.3 to $7 \mathrm{keV} \mathrm{nT}^{-1}$ by this method and constructed a radial dependences of $f^{*}(\mu, L)$, which are shown in Fig. 1. The crosses in Fig. 1 show our calculated points between which the interpolation was performed by the method of least squares.

I have also made the same calculations of the functions $f^{*}(\mu, L)$ for protons with $\alpha_{0}=90^{\circ}$ for Explorer-45 data, averaged in Fritz and Spjeldvik (1981) over 60 orbits for the quiet period 1-15 June 1972. The measurements of proton fluxes on this satellite were carried out at $L<5.25$ in the nine energy channels: 78.6-138.5-195.5-300 keV and 363.5-375-390-430-533-674-872 keV. The results of our calculations of the functions $f^{*}(\mu, L)$ for these data are shown in Fig. 2. To avoid overlapping, the curve for $\mu=0.2 \mathrm{keV} \mathrm{nT}^{-1}$ is raised above the other curves by 1 order of magnitude. As in Fig. 1, crosses in Fig. 2 show our calculated points between which the interpolation was performed by the method of least squares.

In overlapping ranges of $L$ and at the same $\mu$ the results of calculations of the functions $f^{*}(\mu, L)$ for the proton belt, shown in Figs. 1 and 2, are in good agreement with each other, both in shape and in absolute values. A significant difference is obtained only in a narrow interval, at $0.7 \leq \mu \leq 1 \mathrm{keVnT}^{-1}$ on $3 \leq L \leq 4$, where according to Explorer-45, we have flatter spectra and a radial dependence of $f^{*}$. The discrepancy can apparently be related to the solarcyclic variations of the belts, with some geomagnetic activity in November 1977, and to the difference in the averaging of the data of Explorer-45 and ISEE-1.

Positive radial gradients of the functions $f^{*}(\mu, L)$ in Figs. 1 and 2 show that the trapped protons diffuse mainly to the Earth.

\subsection{The calculation of the rates of ionization losses of the trapped protons}

Eq. (1) can be represented as follows:

$$
\frac{\partial}{\partial L}\left(\frac{D_{L L}}{L^{2}} \frac{\partial f^{*}}{\partial L}\right)=\frac{f^{*}}{L^{2} \tau},
$$




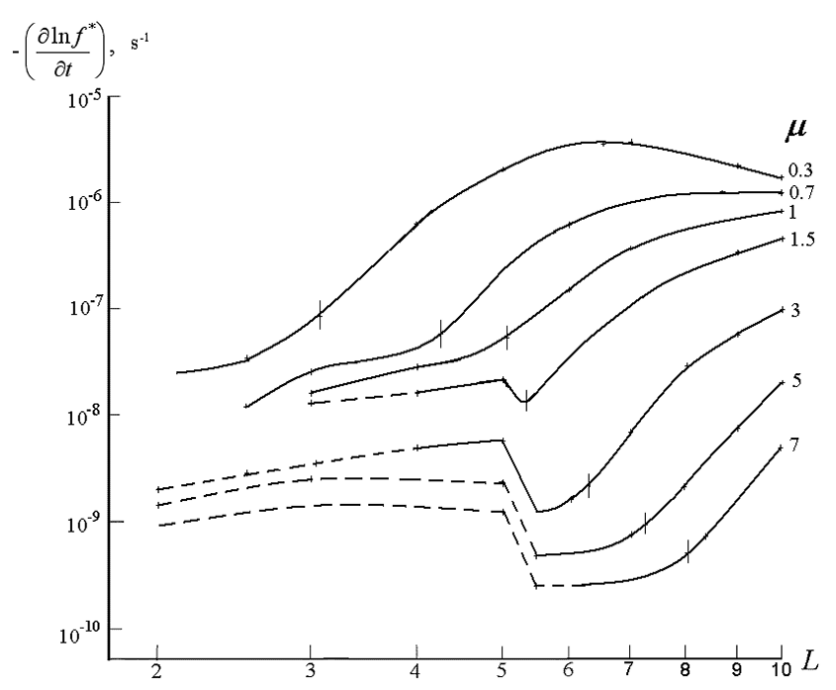

Figure 3. The radial dependence of the rates of the ionization losses of protons with various $\mu$ calculated with regard to the shape of the energy spectra of protons based on the ISEE-1 data from Williams and Frank (1984) for a period of 17 November 1977. Here $\mu$ is given in kiloelectronvolt per nanotesla. The jump on these curves at $L=5.0-5.5$ corresponds to a sharp drop in electron density near the plasmapause. The vertical cuts on these curves mark $L$, at which rate the Coulomb loss is equal to the charge exchange rate of the protons.

where

$\tau^{-1}(\mu, L)=-\frac{1}{f^{*}}\left[\left(\frac{\partial f^{*}}{\partial t}\right)_{\mathrm{cc}}+\left(\frac{\partial f^{*}}{\partial t}\right)_{\mathrm{ce}}\right]=-\frac{\partial \ln f^{*}}{\partial t}$.

Without updating the belts by the radial diffusion, $f^{*}(\mu, L)$ decays exponentially with time constant $\tau(\mu, L)$. I calculate the ionization losses of the protons (Coulomb losses and the losses to charge exchange) on the basis of experimental cross section of charge exchange presented in Claflin (1970) and Lindsay and Stebbings (2005) and on the real experimental spectra of protons (for the same data of the ISEE-1 and Explorer-45).

Coulomb losses and the losses to charge exchange were calculated for the protons with specific values of $\mu$, and then these losses were summed. As a result, I found $L$ dependences of the rates of ionization losses for the protons with different values of $\mu$ (from 0.2 to $7 \mathrm{keV} \mathrm{nT}^{-1}$ ). For these calculations, I used the modern empirical models of the plasmasphere (Østgaard et al., 2003; Zoennchen et al., 2013) and exosphere (Moldwin et al., 2002; Ozhogin et al., 2012). The methodology of these calculations is described in detail in Kovtyukh (2016).

The radial dependences of the rates of the ionization losses of the trapped protons were calculated for 17 November 1977 are shown in Fig. 3. Coulomb losses of protons calculated with regard to the functions $f^{*}(\mu, L)$ for this period (see Fig. 1). They correspond to ISEE-1 data. The dotted plots of these curves result from the extrapolation of the ISEE-1 data

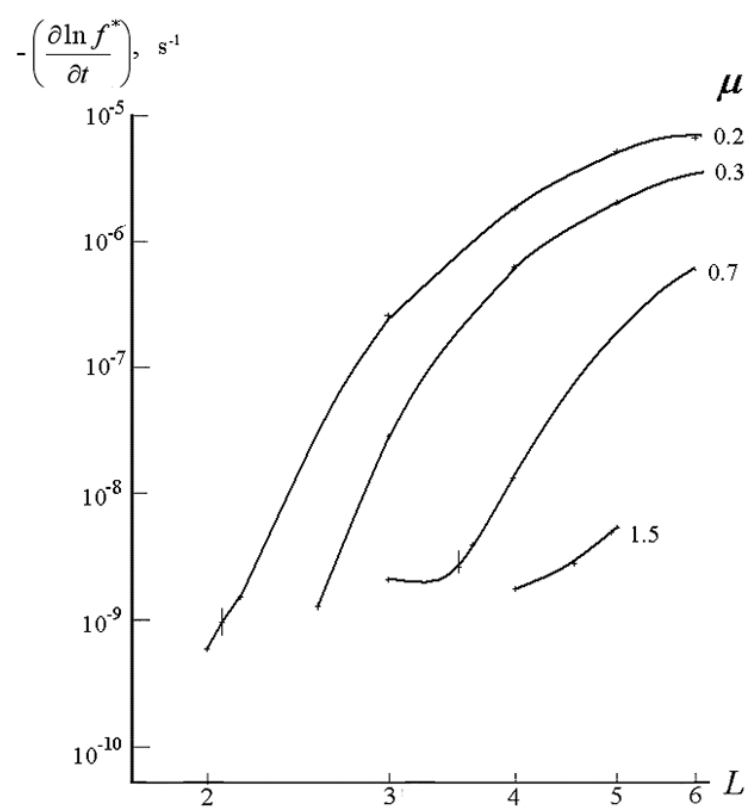

Figure 4. The same as in Fig. 3 for the Explorer-45 data from Fritz and Spjeldvik (1981) for the period of 1-15 June 1972. Here $\mu$ is given in kiloelectronvolt per nanotesla.

on low $L$. For protons with $\mu \geq 1.5 \mathrm{keVnT}^{-1}$, the jump on these curves at $L=5.0-5.5$ reflects a sharp drop in electron density and, as a result, the drop in the rate of the Coulomb losses of protons near the plasmapause.

The vertical cuts on these curves mark $L$, at which rate the Coulomb loss is equal to the charge exchange rate of the protons; i.e., it is the boundary between the small $L$ area dominated by Coulomb losses and the larger $L$ area dominated by the charge exchange loss of protons. The position of this boundary $\left(L_{\mathrm{b}}\right)$ depends on $\mu$ of the protons: $L_{\mathrm{b}} \approx 4.71 \times \mu^{0.32}$, where $\mu$ is given in kiloelectronvolt per nanotesla (Kovtyukh, 2016). This means that $E_{\mathrm{b}} \approx 300 \mathrm{keV}$ and that protons with $E<300 \mathrm{keV}$ dominate the charge exchange with atoms and protons with $E>300 \mathrm{keV}$ dominate the Coulomb losses. At $L \sim 3-10$ this boundary is almost independent of the proton energy. This is mainly due to the fact that the ratio of the density of the electrons of cold plasma to the density of hydrogen atoms does not change very much with changing $L$ (with the exception of the region of the plasmapause).

Radial dependences of the rates of the ionization losses of the trapped protons, calculated for 17 November 1977 (Fig. 3) and 24-25 November 1977 (Fig. 8 in Kovtyukh, 2016), are in good agreement with each other. This is due to the similarity of the shape of the proton spectra in a quiet and a weakly disturbed periods. Some of the differences are associated with slight differences in the spectra of protons measured in these periods, which leads to differences in the rate of the Coulomb losses of protons. 
Figure 4 shows the radial dependence of the rates of ionization losses of the trapped protons calculated for the quiet period 1-15 June 1972. Coulomb losses of protons are calculated with regard to the functions $f^{*}(\mu, L)$ for this period (see Fig. 2). They correspond to Explorer-45 data. From these data, in the region dominated by the Coulomb losses of protons, at $L<4$, the spectra of protons were flatter than the spectra measured on ISEE-1 for the period 17 November 1977, and therefore the losses of protons were less. On another hand, Explorer-45 data, compared to ISEE-1 data, were obtained in a period of higher solar activity; in this period the density of the plasmasphere and exosphere was apparently somewhat higher and, therefore the losses of protons, especially the Coulomb losses at $L<5$, was significantly more (this effect was not considered in our calculations).

In the region of the plasmapause, methodical errors of our calculations of the rates of the Coulomb losses of the trapped protons can be more than in the other regions of the belts. However, from further consideration it will be seen that this circumstance can have an effect only on the calculations of $D_{L L}$ on $5 \leq L \leq 6$ for protons with $\mu \sim 1.5 \mathrm{keV} \mathrm{nT}^{-1}$. For protons with $\mu<1.5 \mathrm{keV} \mathrm{nT}^{-1}$, the charge exchange is dominated in the region of the plasmapause, and for protons with $\mu>1.5 \mathrm{keV} \mathrm{nT}^{-1}$, reliable calculations of $D_{L L}$ can be done only for $L>6$ (for the ISEE-1 data).

\subsection{The calculation of rates of the radial transport of the trapped protons}

We divide the radial dependences of $f^{*}(\mu, L)$ shown in Figs. 1 and 2 into separate segments and integrate Eq. (4) within each such segment taking into account Figs. 3 and 4. As a result, for each value of $\mu$ in Figs. 1 and 2, we obtain the following chain of integrodifferential equations:

$$
\begin{aligned}
& \left.\frac{D_{L L}\left(\mu, L_{i+1}\right)}{L_{i+1}^{2}} \frac{\partial f^{*}}{\partial L}\right|_{L_{i+1}}-\left.\frac{D_{L L}\left(\mu, L_{i}\right)}{L_{i}^{2}} \frac{\partial f^{*}}{\partial L}\right|_{L_{i}} \\
& =\int_{L_{i}}^{L_{i+1}} \frac{f^{*}(\mu, L)}{L^{2} \tau(\mu, L)} \mathrm{d} L,
\end{aligned}
$$

where $L_{i}$ and $L_{i+1}(i=1,2, \ldots, n)$ are the lower and upper boundaries of the corresponding segment of the radial profile $f^{*}(\mu, L)$. After calculating all the derivatives and integrals in Eq. (5), we obtain a system of the linear algebraic equations for a given $\mu$.

The values of the two terms on the left part of Eq. (5) are very close to each other and their difference strongly depends on the radial dependence of $D_{L L}$. In addition, the system of Eq. (5) is incomplete: the number of unknowns $D_{L L}\left(\mu, L_{i}\right)$ is one more than the number of equations. It can be solved only if we exclude one of these unknowns in each system of equations (for each given value of $\mu$ ).

By summing these equations, we exclude from the system of Eq. (5) all intermediate terms, and we get the complete equation. The difference between the normalized diffusion flows on the biggest $L$ and on the smallest $L$ is on the left side of this is, and the normalized integral of the rates of losses of the protons between these extreme $L$ (for a given $\mu$ ) is on the right-hand side.

For general physical reasons, it follows that $D_{L L}$ rapidly decreases with decreasing $L$. This fact is reflected in all the proposed mechanisms of the radial diffusion of particles in the Earth's radiation belts and in the belts of other planets (see, e.g., Kollmann et al., 2011). Primarily, this is due to the fact that the magnetic field increases rapidly with decreasing L.

Therefore, the diffusion flow for the smallest $L$ is much less than for the largest $L$, and we can leave the flow for the largest $L$ on the left side of the complete equation. As a result we obtain a linear equation with one unknown variable and we find from it the value $D_{L L}$ at the external boundary of the $L$ range (for a given $\mu$ ). Substituting this value $D_{L L}$ in system of Eq. (5), we obtain the complete system of equations and gradually find all the other values of $D_{L L}$ at different $L$ (for a given $\mu$ ). Similarly, one can create and resolve the system Eq. (5) for other values of $\mu$. However, I do not want to make any preliminary assumptions about the radial dependence of $D_{L L}$.

For all values of $\mu$ and $L$, shown in Figs. 1 and 2, I calculated $L^{-2}\left(\partial f^{*} / \partial L\right)$ from the left part of Eq. (5). According to our calculations, for protons with $\mu \geq 1.5 \mathrm{keV} \mathrm{nT}^{-1}$ from ISEE-1 data (Fig. 1), the value $L^{-2}\left(\partial f^{*} / \partial L\right)$ monotonically decreases with decreasing $L: 10.4$ times when reducing $L$ from 10 to 4 for $\mu=1.5 \mathrm{keV} \mathrm{nT}^{-1}, 27.3$ times when reducing $L$ from 10 to 5 for $\mu=3 \mathrm{keV} \mathrm{nT}^{-1}, 40.2$ times when reducing $L$ from 10 to 5 for $\mu=5 \mathrm{keV} \mathrm{nT}^{-1}$, and 45.4 times when reducing $L$ from 10 to 6 for $\mu=7 \mathrm{keV} \mathrm{nT}^{-1}$.

So even if we assume that $D_{L L}$ does not depend on $L$, the smaller of the two terms in the left part of the total equations of systems (5), for smallest $L$, can be neglected for protons with $\mu \geq 1.5 \mathrm{keV} \mathrm{nT}^{-1}$. The error of the calculations of $D_{L L}$ at $L=10$, related to this, ranges from $\sim 10 \%$ for $\mu=1.5 \mathrm{keVnT}^{-1}$ to $\sim 2 \%$ for $\mu=7 \mathrm{keVnT}^{-1}$ (if we posit that $D_{L L}$ decreases with decreasing $L$, this error will be much less). Of course, when approaching the lower boundary on $L$ (for a given value of $\mu$ ), the error of our calculations of $D_{L L}$ increased. To this error we must add the errors of calculations $f^{*}$ (Figs. 1 and 2) and the ionization losses of protons (Figs. 3 and 4).

However, for protons with $\mu=1 \mathrm{keVnT}^{-1}$, the value $L^{-2}\left(\partial f^{*} / \partial L\right)$ is reduced only 2.9 times when $L$ is reduced from 9 to 3 . In this case, my method is valid only under the assumption that $D_{L L}$ quite strongly decreases with decreasing $L$. Even more important is the assumption for protons with $\mu<1 \mathrm{keVnT}^{-1}$ for which the dependence $L^{-2}\left(\partial f^{*} / \partial L\right)$ on $L$ is non-monotonic. For protons with $\mu=0.7 \mathrm{keV} \mathrm{nT}^{-1}$, the value of $L^{-2}\left(\partial f^{*} / \partial L\right) \mathrm{de}-$ creases by 10.4 times with $L$ decreasing from 5 to 2.5 , and for protons with $\mu=0.3 \mathrm{keV} \mathrm{nT}^{-1}$, it decreases by 23.4 
times with $L$ decreasing from 4.5 to 2 . However, at $L>5$ for $\mu=0.7 \mathrm{keV} \mathrm{nT}^{-1}$ and at $L>4.5$ for $\mu=0.3 \mathrm{keV} \mathrm{nT}^{-1}$, the value of $L^{-2}\left(\partial f^{*} / \partial L\right)$ increases with decreasing $L$.

Thus, for protons with $\mu<1 \mathrm{keV} \mathrm{nT}^{-1}$, our calculations of $D_{L L}$ according to ISEE-1 data are less reliable than calculations for protons with $\mu \geq 1.5 \mathrm{keV} \mathrm{nT}^{-1}$.

For protons with $\mu<1.5 \mathrm{keVnT}^{-1}$, the ISEE-1 data are well complemented by Explorer-45 data obtained at smaller $L$. According to the Explorer-45 data (Fig. 2), the value of $L^{-2}\left(\partial f^{*} / \partial L\right)$ monotonically decreases with decreasing $L$ for protons with $\mu=0.2-3 \mathrm{keV} \mathrm{nT}^{-1}: 54.4$ times when reducing $L$ from 4 to 2 for $\mu=0.2 \mathrm{keV} \mathrm{nT}^{-1}, 8.6$ times when reducing $L$ from 4.5 to 2.5 for $\mu=0.3 \mathrm{keV} \mathrm{nT}^{-1}, 1.6$ times when reducing $L$ from 4 to 3 for $\mu=0.7 \mathrm{keV} \mathrm{nT}^{-1}, 4.5$ times when reducing $L$ from 5 to 4 for $\mu=1.5 \mathrm{keVnT}^{-1}$, and 1.6 times when reducing $L$ from 5 to 4.5 for $\mu=3 \mathrm{keV} \mathrm{nT}^{-1}$. Therefore, this method appears to be applicable to Explorer45 data for protons with $\mu=0.2-0.7 \mathrm{keV} \mathrm{nT}^{-1}$.

In calculating the derivatives on the left-hand side and integrals on the right-hand side of Eq. (5), I divided the scale of $L$ into fairly short intervals where the functions $f^{*}(\mu, L)$ and $\tau(\mu, L)$ are well approximated by a power law with different exponents. All the approximation functions were joined together at the boundaries of these intervals.

The results of our calculations of $D_{L L}(\mu, L)$ based on Figs. 1 and 3 (ISEE-1) are shown in Fig. 5, and those based on Figs. 2 and 4 (Explorer-45) are shown in Fig. 6. The numbers on the right-hand side of Figs. 5 and 6 refer to the values of $\mu$ (in kiloelectronvolt per nanotesla).

From Figs. 5 and 6, we see that the results of our calculations of $D_{L L}(\mu, L)$ based on the data from ISEE-1 and Explorer-45 are in good agreement with each other for $\mu=0.3 \mathrm{keV} \mathrm{nT}^{-1}$ (they differ by no more than $\sim 2.5$ times their value). For protons with $\mu=0.7 \mathrm{keV} \mathrm{nT}^{-1}$, the functions of $D_{L L}(L)$ are sewn together well at $L=4$. For protons with $0.7<\mu<1 \mathrm{keV} \mathrm{nT}^{-1}$ in the region $3 \leq L \leq 4$, where according to ISEE-1 and Explorer-45 (see Figs. 1 and 2) the radial gradients of the functions $f^{*}(\mu, L)$ are significantly different, good agreement was also obtained between the calculated values of $D_{L L}(\mu, L)$. However, for $\mu=1.5 \mathrm{keV} \mathrm{nT}^{-1}$ the values of $D_{L L}$ calculated at $L=4.5-5.0$ on the basis of the Explorer-45 data were $\sim 7-8$ times smaller than the values calculated with the ISEE-1 data.

This discrepancy is reduced if we consider that the data from Explorer-45 are obtained in a period of higher solar activity. In this period the density of the plasmasphere and exosphere was apparently higher than during the period of measurements on ISEE-1. Therefore, the losses of protons were greater than our calculated values, especially at $L<5$. In this regard, the values of $D_{L L}$ shown in Fig. 6 should be increased (see Eqs. 4 and 5).

The errors of my method of calculating $D_{L L}$ depend on the width of the range of $L$ in which we have conducted the calculations: the narrower this range, the more errors there are in our calculations. For different $\mu$ the width of the range

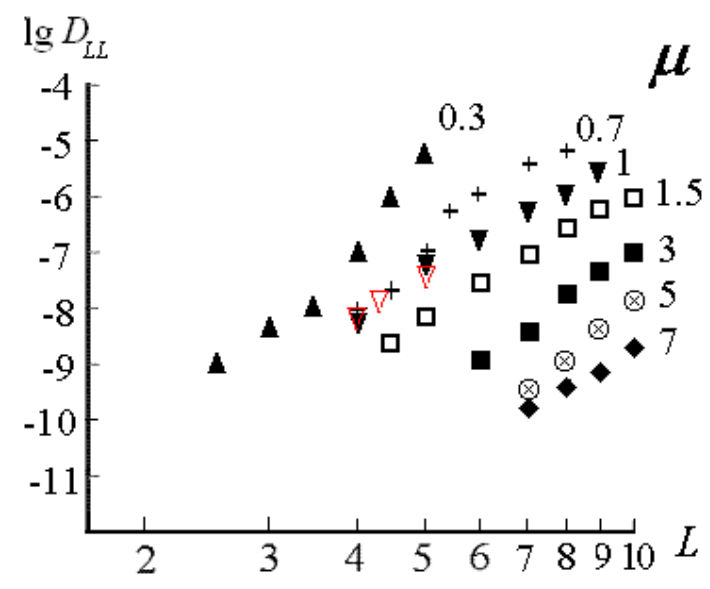

Figure 5. The results of calculations of the values of $D_{L L}(\mu, L)$ near the equatorial plane based on the ISEE-1 data in Williams and Frank (1984) for the weakly disturbed period from 17:52 to 21:05 UT, 17 November 1977. In this period $\mathrm{Kp}=1_{-}\left(\mathrm{Kp} \leq 2_{+}\right.$ for $12 \mathrm{~h}$ prior to this period UT). These results take into account Figs. 1 and 3. Red signs show values of $D_{L L}$ for protons with $\mu=1 \mathrm{keV} \mathrm{nT}^{-1}$, calculated according to ISEE-1 data in Williams (1981) for the quiet period of 24-25 November 1977 $(\mathrm{Kp} \leq 1)$. Here, $D_{L L}$ is given in values per second and $\mu$ is given in kiloelectronvolt per nanotesla.

$L$ is different. For sufficiently long series of calculations (for large numbers of Eq. 5), when the maximum (at the upper limit of the range $L$ ) and the minimum (at the lower end of this range) values of $D_{L L}$ differ by more than 1 order of magnitude, i.e., for $\mu$ from 0.3 to $5 \mathrm{keV} \mathrm{nT}^{-1}$ based on the ISEE1 data and for $\mu \sim 0.2 \mathrm{keV} \mathrm{nT}^{-1}$ based on the Explorer-45 data, errors of our calculations of $D_{L L}$ do not exceed $10 \%$ at large $L$. With decreasing $L$ and with increasing $\mu$ of protons, these errors increase to some tens of percent.

Since the Explorer-45 data are limited to a maximum available $L \sim 5$, correct calculations of $D_{L L}$ from these data are possible only for $\mu<1 \mathrm{keVnT}^{-1}$. For large values of $\mu$ the ranks of our calculations of $D_{L L}$ on the scale of $L$ are short, which leads to large methodical errors and to a significant underestimation of $D_{L L}$ in the calculations for protons with $\mu>1 \mathrm{keV} \mathrm{nT}^{-1}$ based on the Explorer- 45 data.

For $L>5$ the magnetosphere is asymmetric in magnetic local time (MLT), and with the growth of $L$ this asymmetry increases. Because in the quiet periods the asymmetry of the magnetosphere for $5<L<10$ is not very large and the function $f(\mu, L)$ in Eq. (1) as the function $f^{*}(\mu, L)$ in Eq. (5) is averaged over the drift of particles around the Earth, the average values of $L$ are close to the values given in Fig. 5 . According to our estimates, the associated error does not exceed other methodical errors in our calculations.

The transition from a dipole model to a more realistic mathematical model of the geomagnetic field leads to some changes in the calculated values of $D_{L L}(\mu, L)$. However, the experimental data used here were obtained in quiet and 


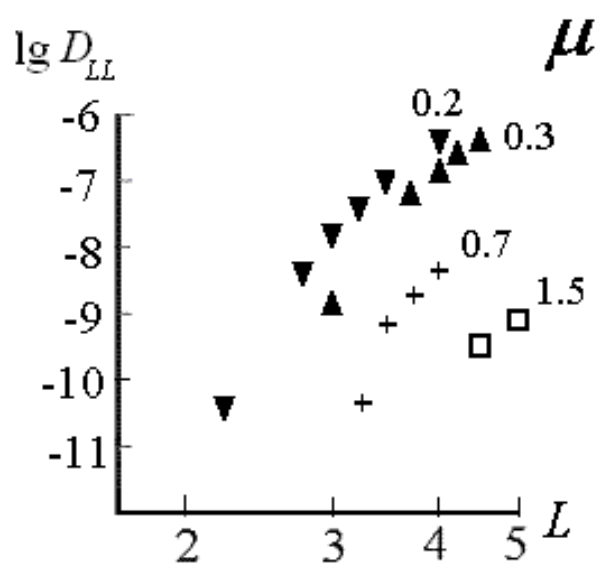

Figure 6. The results of calculations of the values of $D_{L L}(\mu, L)$ near the equatorial plane based on the averaged Explorer-45 data in Fritz and Spjeldvik (1981) for the quiet period of 1-15 June 1972. These results take into account Figs. 2 and 4. Here $D_{L L}$ is given in values per second and $\mu$ is given in kiloelectronvolt per nanotesla.

slightly disturbed $(\mathrm{Kp}<2)$ periods. The ISEE-1 data relate to near-noon sector of MLT, and the Explorer-45 data were obtained at $L<5$. So, we can hope that the deviations of the geomagnetic field from the dipole configuration in the outer regions of the trap will not lead to significant changes in the calculated values of $D_{L L}(\mu, L)$, and our main conclusions will not change.

Taking into account all possible errors, the calculated values of $D_{L L}(\mu, L)$ are shown in Figs. 5 and 6. These deviate from the real values by no more than $\sim 2.5$ times their own value and, as a rule, do not exceed the size of the symbols in Figs. 5 and 6 (for all values of $L$ and $\mu$ of protons considered here, except for $\mu=1.5 \mathrm{keV} \mathrm{nT}^{-1}$ in Fig. 6).

This is confirmed by a comparison between our calculations according to data from ISEE-1 for the period 17 November 1977 and according to those from 24-25 November 1977. For these periods, we obtained values of $D_{L L}(\mu, L)$ that were close to each other at the same values of $\mu$ and $L$. For protons with $0.5 \leq \mu \leq 3 \mathrm{keV} \mathrm{nT}^{-1}$, they differ by no more than 1.5-2.0 times their own value. For comparison, red signs in Fig. 5 show several values of $D_{L L}$ for protons with $\mu=1 \mathrm{keV} \mathrm{nT}^{-1}$, calculated according to ISEE-1 data (Williams, 1981) for the quiet period of 24-25 November $1977(\mathrm{Kp} \leq 1)$.

In the total errors of our calculations of $D_{L L}$, the errors associated with models of the plasmasphere (see Kovtyukh, 2016) play a major role. To this rather large value (up to $\sim 2.5$ times) much smaller errors of the measurements of proton fluxes based on ISEE-1 and Explorer-45 and methodical errors (see above) are added. Some errors can also be added by physical processes unaccounted for here, such as plasma instability and the interaction of protons with electromagnetic waves and micro-injections of hot plasma from the tail of the magnetosphere (for low-energy protons at large $L$ ). How- ever, our calculations are carried out for quiet and weakly disturbed periods in Earth's magnetosphere when the plasma distribution is stable, and fast dynamic processes can be neglected in comparison with radial diffusion.

\section{Discussion}

It has been shown that at $L>3$, all stationary distributions (spatial, energy and pitch angle) of protons (and other ions) of the Earth's radiation belts are interrelated and should be formed by mechanisms which provide the radial transport of these particles while conserving $\mu$ and $K$. Kovtyukh (1984, 1985a, b, 1989, 1994, 1999a, b, 2001) has given the fullest and most comprehensive justification of this interrelation in the distributions of protons (and other ions) as a result of the data analysis of 22 missions (Explorer12, Explorer-14, Mariner-4, Explorer-33, European Space Research Organisation satellite 2 (ESRO-2), Injun-4, Injun5, 1968-26B, Orbiting Vehicle 1-19 (OV1-19), Explorer45, 1972-076B, Molnija-1, Applications Technology Satellite 6 (ATS-6), Molnija-2, ISEE-1, Spacecraft Charging at High Altitudes (SCATHA), AMPTE/Charge Composition Explorer (CCE), Gorizont-21, Akebono, CRRES, Gorizont35 and the Engineering Test Satellite VI (ETS-VI)) for 34 years of space research (1961-1994). For protons with $\mu>0.5 \pm 0.2 \mathrm{keVnT}^{-1}$, such a situation can only be provided by mechanisms of the radial diffusion of particles to the Earth from the outer boundary of belts while conserving $\mu$ and $K$ (Kovtyukh, 2001).

The main result of our calculations is the strong dependence of $D_{L L}$ not only on $L$ but also on $\mu$. Figures 5 and 6 show that for all considered $L$, the values of $D_{L L}$ decrease rapidly with increasing values of $\mu$.

This result can be seen from Figs. 1-4 and Eq. (4) before $D_{L L}(\mu, L)$ is calculated: for any given $L$, the rates of the ionization losses of protons decrease with increasing $\mu$ (see Figs. 3 and 4), but the value $\left(\partial \ln f^{*} / \partial L\right)$ increases or remains almost unchanged (see Figs. 1 and 2). Therefore, to keep the balance of radial diffusion and loss of particles, the coefficient of $D_{L L}$ should decrease with $\mu$ increasing. If other possible losses (primarily, the interaction of protons with the waves) are take into account, the dependence of $D_{L L}$ on $\mu$ only increases.

The effect of reducing $D_{L L}$ with increasing $\mu$ is clearly expressed in the ISEE-1 data presented in Fig. 2a in Williams (1981). From this figure, it is seen that the radial gradient of $f(\mu, L)$ increases sharply in the transition from high to low $L$. The greater $\mu$ is, the more $L$ there is, where this happens. This effect indicates a decrease in $D_{L L}$ with $\mu$ increasing. However, this effect was not discussed in Williams (1981). It clearly contradicts the theory of the radial transport of trapped particles under the influence of sudden impulses (SIs) of the magnetic field, which was dominant at the time when Williams was writing. 
The mechanism of particle transport under the influence of SI was proposed by Kellogg (1959), and in many works it has been used as the main mechanism. It is implemented when fluctuations in the dynamic pressure of the solar wind influence the magnetosphere and is usually called magnetic diffusion. I denote the diffusion coefficient for this mechanism by $D_{L L}^{\mathrm{M}}$. Models of the Earth's radiation belts based on the mechanism of magnetic diffusion were created by Nakada and Mead (1965) and Tverskoy $(1965,1969)$. In the model of Nakada and Mead (1965), $D_{L L}^{\mathrm{M}}=2.3 \times 10^{-15} \times L^{10} \mathrm{~s}^{-1}$. In the model of Tverskoy $(1965,1969), D_{L L}^{\mathrm{M}}=5 \times 10^{-14} \times$ $L^{10} \mathrm{~s}^{-1}$. In these models it is supposed that the spectrum of magnetic fluctuations has a power-law form with an exponent of -2 ; in this case $D_{L L}^{\mathrm{M}}$ does not depend on the drift frequency of the particles or their energy and $\mu$ but only on L.

The mechanism of magnetic diffusion is efficient only for traps with a strong azimuthal asymmetry of the geomagnetic field. But in the depths of the geomagnetic trap the magnetic field is almost symmetric and, therefore, the efficiency of the magnetic diffusion should be very small.

Another popular mechanism of radial transport of trapped particles is their diffusion under the action of the fluctuations of an electric field in the magnetosphere during substorms (Fälthammar, 1965, 1966, 1968; Cornwall, 1968, 1972). In contrast to the vortex electric fields generated in the magnetosphere during SI, the electric field of a substorm can be described with an electric potential, and such a mechanism of particle transport is usually called electric diffusion. It does not depend on the azimuthal asymmetry of the magnetic field. In this mechanism $D_{L L}$ depends on $\mu$ and on the charge of the particles. I denote the diffusion coefficient for this mechanism by $D_{L L}^{E}$. According to Cornwall (1968, 1972), for protons

$D_{L L}^{E}=\left(1.5 \times 10^{-10}-1.5 \times 10^{-9}\right) \frac{L^{10}}{L^{4}+\mu^{2}}$,

where $D_{L L}^{E}$ is measured as values per second and $\mu$ is measured in megaelectronvolt per gauss. For $\mu>5 \mathrm{keV} \mathrm{nT}^{-1}$ $\left(>500 \mathrm{MeVG}^{-1}\right)$ at $L>5, \mu^{2} \gg L^{4}$ and, according to Eq. (6), $D_{L L}^{E} \propto \mu^{-2} L^{10}$, but for lower values of $\mu$, the dependence of $D_{L L}^{E}$ on $L$ is weakened at large $L$.

Equation (6) for $D_{L L}^{E}$ and the expression for $D_{L L}^{\mathrm{M}}$ were parameterized for Kp by Brautigam and Albert (2000). With $\mathrm{Kp}=1$ the expression for $D_{L L}^{E}$ in Brautigam and Albert (2000) corresponds to Eq. (6) with the coefficient on the right-hand side of the expression $\sim 9 \times 10^{-10}$, and $D_{L L}^{\mathrm{M}} \sim 1.8 \times 10^{-14} \times L^{10} \mathrm{~s}^{-1}$; i.e., they correspond to the average of $D_{L L}^{\mathrm{M}}$ given by Nakada and Mead (1965) and Tverskoy (1969). According to Brautigam and Albert (2000), with Kp increasing from 1 to 6 , the values of $D_{L L}^{E}$ increase $\sim 200$ times and the values of $D_{L L}^{\mathrm{M}}$ increase $\sim 340$ times.

The functions of $D_{L L}^{\mathrm{M}}$ and $D_{L L}^{E}$ depend on $L$ and $\mu$ in different ways, and therefore in different regions of $\{L, \mu\}$, space their ratio is different. As $D_{L L}^{E}$ depends on $\mu$ and decreases with decreasing $L$ to less than $D_{L L}^{\mathrm{M}}$, if $D_{L L}^{\mathrm{M}}$ dominates at large $L, D_{L L}^{E}$ can dominate at small $L$. If $D_{L L}^{E}$ dominates for small $\mu, D_{L L}^{\mathrm{M}}$ can dominate for large $\mu$. In addition, this ratio can change depending on magnetic activity.

These circumstances lead to different conclusions for the ratio of $D_{L L}^{\mathrm{M}}$ to $D_{L L}^{E}$. The conclusions were drawn in different articles and are sometimes incompatible with each other. So, for electrons with $\mu=5-50 \mathrm{keV} \mathrm{nT}^{-1}$ and related fluctuations of the electric field at $L=3-7$, it has been argued based on CRRES data that $D_{L L}^{E} \ll D_{L L}^{\mathrm{M}}$ (Brautigam et al., $2005)$, but based on the fluctuations of the magnetic field at ground stations and based on AMPTE and GOES data, it has been argued, for the same $L$ and for the same $\mu$ of electrons, that $D_{L L}^{\mathrm{M}} \ll D_{L L}^{E}$ (Ozeke et al., 2012) for both $\mathrm{Kp}=1$ and $\mathrm{Kp}=6$ (see Fig. 11 in Ozeke et al., 2012). In Ozeke et al. (2014), the conclusions of Ozeke et al. (2012) were supported by the analysis of data from CRRES and GOES, and another parameterization, different from that of Brautigam and Albert (2000), was proposed for $D_{L L}^{E}$ and $D_{L L}^{\mathrm{M}}$ for Kp (and $L$ ) where these parameters do not depend on $\mu$.

In many works, $D_{L L}^{\mathrm{M}}$ and $D_{L L}^{E}$ have been considered to be modes of radial diffusion that are independent of each other. In our calculations of $D_{L L}$, I only used data for the particles and did not carry out a separation of $D_{L L}$ for different modes. In the course of further discussion it will be shown that the function of $D_{L L}(\mu, L)$ calculated here corresponds to the uniform diffusion mode which operates in a broad band on $L$ and the energies of protons.

The values of $D_{L L}$ are determined by the spectral density of the fluctuations (pulsations) of the electric and magnetic fields (Schulz and Lanzerotti, 1974). In theoretical works a power dependence of $D_{L L}$ on $L$ is usually postulated: $D_{L L} \propto L^{n}$. This corresponds to the power spectra of the fluctuations of the fields. For different mechanisms of the radial diffusion of the particles, the parameter $n$ takes different values. Therefore, in sufficiently wide ranges of $L$, the dependence of $D_{L L}$ on $L$ for particles with a fixed $\mu$ is not described by a simple power law. This is evident in the evaluation of the parameter $n$, obtained from the experimental data: the parameter $n$ takes significantly different values in different intervals of $L$ and $\mu$ (e.g., Schulz and Lanzerotti, 1974). According to Fig. 5 , in the range of $\mu \sim 1-7 \mathrm{keV} \mathrm{nT}^{-1}$, values of the parameter $n \sim 7.5,7.4,8.2,10$ and 7.7 for $\mu \sim 1$, $1.5,3,5$ and $7 \mathrm{keV} \mathrm{nT}^{-1}$ (average $n \sim 8.2$ ).

Figure 7 presents the dependence of $D_{L L}(\mu)$ on $L=7$ based on the results shown in Fig. 5. Here $D_{L L}$ is given in values per second and $\mu$ is given in kiloelectronvolt per nanotesla. This dependence is shown by a thick curve, and the signs on it correspond to the signs in Fig. 5. In addition, in Fig. 7 thin lines with dots show the dependences of $D_{L L}(\mu)$ on $L=7$ for electric diffusion, which are calculated according to Eq. (6). Thin horizontal lines show $D_{L L}$ at $L=7$ for 


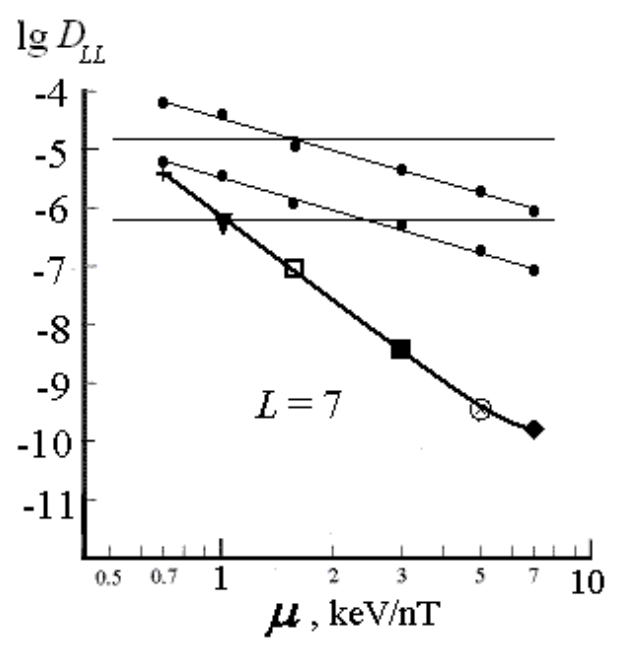

Figure 7. The dependence of the $D_{L L}$ on $\mu$ for $L=7$. The thick curve corresponds to the results given in Fig. 5. The thin curves correspond to Eq. (6) for the electric diffusion. The lower horizontal line is the value of $D_{L L}$ in Nakada and Mead (1965), and the upper line is the value of $D_{L L}$ in Tverskoy $(1965,1969)$. Here $D_{L L}$ is given in values per second.

magnetic diffusion, as given in Nakada and Mead (1965) and Tverskoy $(1965,1969)$.

Figure 7 shows that the results of our calculations are not consistent, not only with magnetic diffusion but also with electric diffusion, as described by Eq. (6). Different linear combinations of $D_{L L}^{\mathrm{M}}$ and $D_{L L}^{E}$ do not lead to reasonable agreement between these results and our calculations.

Figure 8 presents the dependences of $D_{L L}(\mu)$ on different $L$, in the range from 4 to 10 , based on the results shown in Fig. 5. Here, as in Fig. 7, $D_{L L}$ is given in values per second and $\mu$ is given in kiloelectronvolt per nanotesla. The thick lines in this figure are calculated using the method of least squares. The numbers near these lines correspond to the values of $L$.

According to Fig. 8, $D_{L L}(\mu) \propto \mu^{-m}$, where the parameter $m$ depends on $L$. The maximum value of $m(\sim 4.4-4.5)$ is achieved at $L \sim 6-7$; the minimum $m(\sim 2.4)$ is achieved at $L=4$. The value of this parameter averaged over $L$ equals 3.9 (or 4.1 if excluding the value of $m$ for $L=4$ ).

The results of our calculations of $D_{L L}$, shown in Figs. 5 and 8 , for protons with $\mu$ from $\sim 0.7$ to $\sim 7 \mathrm{keV} \mathrm{nT}^{-1}$ at $L \approx 4.5-10$, are most adequately described by the following expressions:

$D_{L L}(\mu, L) \approx 4.9 \times 10^{-14} \mu^{-4.1} L^{8.2}$,

where $D_{L L}$ is measured in values per second and $\mu$ is measured in kiloelectronvolt per nanotesla. Replacing $\mu$ by $E$ in Eq. (7), we get

$D_{L L}(E, L) \approx 1.3 \times 10^{5}(E L)^{-4.1}$,

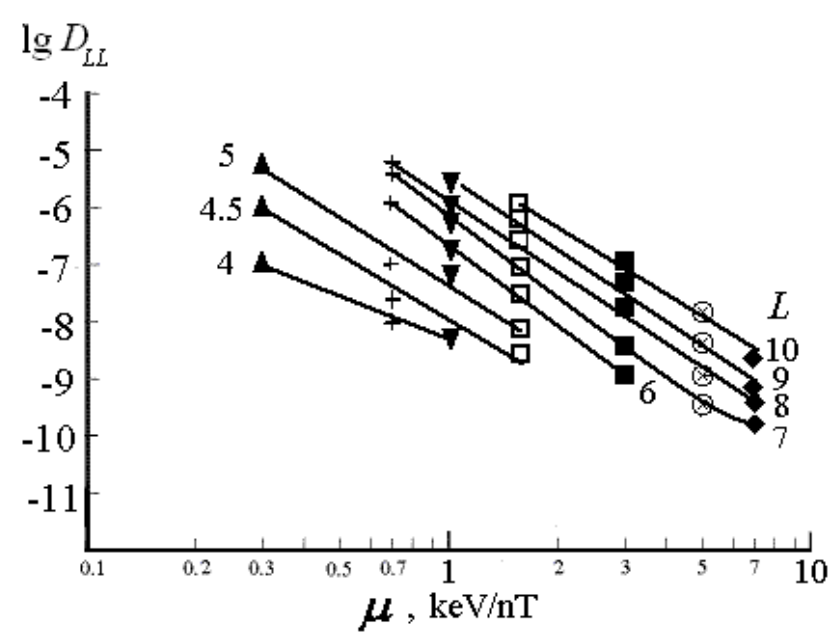

Figure 8. The dependences of $D_{L L}(\mu)$ on different $L$ (in the range from 4 to 10), which corresponds to the results shown in Fig. 5. Here $D_{L L}$ is given in values per second.

where $D_{L L}$ is measured in values per second and $E$ is measured in kiloelectronvolt. Replacing $E L$ by drift frequency in Eq. (8), we get

$D_{L L}\left(f_{\mathrm{d}}\right) \approx 1.2 \times 10^{-9} f_{\mathrm{d}}^{-4.1}$,

where $f_{\mathrm{d}}$ is the azimuthal drift frequency of nonrelativistic protons (inverse value of the drift period of a particle around the Earth), $f_{\mathrm{d}}$ is measured in millihertz and $D_{L L}$ is measured in values per second.

In Eqs. (7)-(9) the results of the calculations of $D_{L L}$ for protons with $\mu=0.3 \mathrm{keVnT}^{-1}$ are not taken into account. These differ greatly (see Fig. 5) from the results of the calculations for protons with $\mu \geq 0.7 \mathrm{keV} \mathrm{nT}^{-1}$. They correspond to the low-energy part of the spectra of protons, and even in quiet periods, this part of the spectra of trapped particles is very sensitive to the cyclotron and other plasma instabilities that were not taken into account in our calculations.

The Eqs. (7)-(9) contradict the theory of diffusion under the action of SI (magnetic diffusion). According to this theory, $D_{L L}$ does not depend on the $\mu, E$ and $f_{\mathrm{d}}$ of particles (Nakada and Mead, 1965; Tverskoy, 1965, 1969; Schulz and Lanzerotti, 1974).

In the dipole approximation, the azimuthal drift frequency of nonrelativistic trapped particles is $f_{\mathrm{d}}=11.8 \times \mu L^{-2}$, where $f_{\mathrm{d}}$ is given in millihertz and $\mu$ is given in kiloelectronvolt per nanotesla. The results for protons with $\mu=0.3$, $0.7,1,1.5,3,5$ and $7 \mathrm{keV} \mathrm{nT}^{-1}$ shown in Fig. 5 correspond to $f_{\mathrm{d}} \sim 0.14-0.57,0.13-0.52,0.15-0.74,0.18-0.87,0.35-0.98$, $0.59-1.20$ and $0.83-1.68 \mathrm{mHz}$. These frequencies belong to the range of Pc6.

For magnetic field fluctuations $D_{L L} \propto f_{\mathrm{d}}^{2} P_{\mathrm{M}}\left(f_{\mathrm{d}}\right) L^{10}$ (Fälthammar, 1965; Nakada and Mead, 1965; Tverskoy, 1965), and for electric field fluctuations $D_{L L} \propto P_{\mathrm{E}}\left(f_{\mathrm{d}}\right) L^{6}$ (Fälthammar, 1966, 1968), where $P_{\mathrm{M}}$ and $P_{\mathrm{E}}$ represent the 
spectral density of these fluctuations (pulsations). Therefore, to satisfy the Eqs. (7)-(9), the spectrum of magnetic field fluctuations should have an exponent of about -6 and should be attenuated with increasing $L$ as $L^{-10}$. The spectrum of electric field fluctuations should have an exponent of about -4 and should be attenuated with increasing $L$ as $L^{-6}$.

Unfortunately, in the range of $f<1 \mathrm{mHz}$ the spectra of fluctuations (pulsations) of the electric and magnetic fields in the geomagnetosphere and in the surrounding area are insufficiently studied. In the range of $0.1-1.7 \mathrm{mHz}$ (Pc6) spectra of these fluctuations are irregular and vary strongly. Compared to higher-frequency ranges, there were few reliable measurements of the pulsations of the electric and magnetic fields in this interval until the recent publication of the results from experiments on THEMIS.

According to the measurements of the Geotail and Wind satellites in the near-Earth foreshock (Berdichevsky et al., 1999), typical spectra of the magnetic field fluctuations in the range of $\sim 0.5-100 \mathrm{mHz}$ can be approximated by a power law with an exponent of -4 to -2 . In the upper part of this range $(>10 \mathrm{mHz})$, some of these spectra can be approximated by a power law with an exponent of -6 , but at the bottom of the range $(<10 \mathrm{mHz})$ the spectrum is flatter and the exponent is close to the value -2 (see Fig. 8 in this article). This is confirmed by CRRES data (Ali et al., 2015): in the range of $\sim 1-8 \mathrm{mHz}$ averaged spectra of the magnetic field fluctuations are very hard and almost flat.

In the range of $1-100 \mathrm{mHz}$ the spectra of the fluctuations of the magnetic field were also measured at ground stations associated with cusp/cleft (Posch et al., 1999). These spectra are irregular and vary strongly depending on the speed of the solar wind. For a power-law approximation, the average exponent of these spectra is close to the value -4 in the range of $10-100 \mathrm{mHz}$, but in the lower part of the range $(1-10 \mathrm{mHz})$ an exponent of the spectral density of these fluctuations is close to $-5 / 3$ (see Fig. 6 in this article).

According to GOES (on geosynchronous orbit) and Wind (in the solar wind) in the range of $0.2-1.7 \mathrm{mHz}$ (Kepko and Spence, 2003), the amplitude spectra of magnetic fluctuations are irregular (fine structure with narrow peaks), but the average amplitude of these fluctuations decreases with increasing frequency by a power law with an exponent of -1.8 to -1.5 (see Figs. 4, 9, 11, 13 and 15 in this article); i.e., for the average spectral density we have an exponent of -4.6 to -4.0 .

Thus, the experimental spectra of the magnetic field fluctuations (pulsations) are not consistent with Eqs. (7)-(9) obtained here.

On the basis of over 7 years of averaged data from THEMIS for all MLT, for different Kp (from 0 to 5) and for different $L$ (from 3.5 to 7.5), Liu et al. (2016) constructed the spectra of electric field fluctuations (pulsations) in the range of $\sim 0.5-10 \mathrm{mHz}$ (unfortunately, the data at $f<0.5 \mathrm{mHz}$ are not given). In the range of $\sim 0.5-2 \mathrm{mHz}$ these spectra are very soft, especially during quiet and slightly disturbed
$(\mathrm{Kp}=0-2)$ periods. For $\mathrm{Kp}=0-2$, according to Fig. 2 from Liu et al. (2016), in the range of $\sim 0.5-2 \mathrm{mHz}$ the dependence of the spectral density of the fluctuations on the frequency can be approximated by a power law with an exponent that varies from $-(3.3-3.9)$ at $L>5.5$ to -5.4 at $L=4.5$ and -10.5 at $L=3.5$; i.e., at $L \geq 4.5$ the average value of this exponent is close to -4 . Our calculations also show that the slope of the radial dependence of $D_{L L}(\mu, L)$ usually increases with decreasing $L$ (see Figs. 5 and 6).

According to Fig. 2 in Liu et al. (2016), at a frequency of $\sim 0.5 \mathrm{mHz}$ the spectral density of the electric field fluctuations decreases also with increasing $L$ (approximately as $L^{k}$, where mean square value of the exponent $k$ is changed from -6.5 when $\mathrm{Kp}=0$ to -7.3 when $\mathrm{Kp}=2$ ). But already at a frequency of $\sim 1.0 \mathrm{mHz}$ this spectral density does not depend on $L$, and at frequencies of $\sim 1$ to $10 \mathrm{mHz}$ this value increases with $L$.

Thus, according to THEMIS data averaged over 7 years for all MLT, for periods with $\mathrm{Kp}=0-2$ the spectral density of the electric field fluctuations (pulsations) at $L \geq 4.5$ for $f \sim 0.5-1 \mathrm{mHz}$ can be described by the following expression: $P_{\mathrm{E}} \propto f_{\mathrm{d}}^{-4} L^{-6}$. Substituting this expression into the formula $D_{L L} \propto P_{\mathrm{E}}\left(f_{\mathrm{d}}\right) L^{6}$ (Fälthammar, 1966, 1968) for the radial diffusion of particles influenced by the electric field fluctuations, we obtain $D_{L L} \propto f_{\mathrm{d}}^{-4}$. This result corresponds to our Eq. (9), and, hence, it is consistent with Eq. (8) and Eq. (7), which refer to the main cluster of calculated points in Fig. 5 and satisfy the ranges $f_{\mathrm{d}} \sim 0.5-1.2 \mathrm{mHz}$ (Pc6) and $\mathrm{Kp}=0-2$.

On the basis of the spectra of electric field fluctuations, Liu et al. (2016) calculated $D_{L L}$ for relativistic electrons with $\mu=5-40 \mathrm{keVnT}^{-1}\left(500-4000 \mathrm{MeV} \times \mathrm{G}^{-1}\right)$ at $L=3.5-7.5$. The drift frequency of these electrons corresponds to the range $f_{\mathrm{d}} \sim 1-4 \mathrm{mHz}$ (Pc5), where the spectrum of fluctuations is flatter and the spectral density increases with $L$. For these reasons, in comparison to our calculations for protons for a range $f_{\mathrm{d}} \sim 0.15-1.2 \mathrm{mHz}$ (Pc6), in Liu et al. (2016) the dependence of $D_{L L}$ on $\mu$ is much weaker and the dependence of $D_{L L}$ on $L$ is slightly stronger.

Note that for electrons with $\mu=1-50 \mathrm{keVnT}^{-1}$ in the range of $L=3-7$, the functions of $D_{L L}$ are calculated also for the spectra of electric field fluctuations in the range of $0.2-15.9 \mathrm{mHz}$ measured by CRRES (Brautigam et al., 2005). For $\mathrm{Kp}=1$, about the same dependence of $D_{L L}$ on $L$ is obtained as in Eq. (7) $\left(D_{L L} \propto L^{8}\right)$, but the dependence of $D_{L L}$ on $\mu$ was much weaker (see Fig. 9 in Brautigam et al., 2005).

\section{Conclusion}

I calculate the value $D_{L L}$, which is a measure of the rate of the radial transport (diffusion) of the particles of the Earth's radiation belts, directly from data on the fluxes and energy spectra of protons with an equatorial pitch angle of $\alpha_{0} \approx 90^{\circ}$ during quiet and slightly disturbed $(\mathrm{Kp} \leq 2)$ periods. 
This is done by successively solving the equations of the balance of the radial transport/acceleration and ionization losses of protons for the stationary belt. Calculations of the ionization losses of protons (Coulomb losses and charge exchange) were carried out on the basis of modern models of the plasmasphere and the exosphere.

To find $D_{L L}$ I calculated the radial dependences of the distribution function $f^{*}(L, \mu)$ for different $\mu$. For each of the given values of $\mu$, these dependences were divided into short segments and the systems (chains) of integrodifferential equations describing the balance of radial transport and losses of protons were solved. This work is carried out here for the first time.

For these calculations I used the data of ISEE-1 for protons with an energy of 24 to $2081 \mathrm{keV}$ at $L \approx 2-10$ and the data of Explorer-45 for protons with an energy of 78.6 to $872 \mathrm{keV}$ at $L \approx 2-5$. The values of $D_{L L}$ calculated from the data of ISEE1 and Explorer-45, in the overlapping intervals of $L$ and $\mu$, are in good agreement with each other.

As a result of the calculations, I found that in the range of $L \approx 2.5-10$ the dependences of $D_{L L}(L)$ are significantly different for protons with different $\mu$ (from 0.2 to $7 \mathrm{keV} \mathrm{nT}^{-1}$ ). The values of $D_{L L}$ decrease rapidly with decreasing $L$ as well as with increasing $\mu$.

It is shown that for protons with $\mu$ from $\sim 0.7$ to $\sim 7 \mathrm{keV} \mathrm{nT}^{-1}$ at $L \approx 4.5-10$, the functions of $D_{L L}$ can be approximated by the following equivalent expressions: $D_{L L} \approx$ $4.9 \times 10^{-14} \mu^{-4.1} L^{8.2}$, or $D_{L L} \approx 1.3 \times 10^{5}(E L)^{-4.1}$, or $D_{L L} \approx 1.2 \times 10^{-9} f_{\mathrm{d}}^{-4.1}$, where $f_{\mathrm{d}}$ is the drift frequency of the protons (in $\mathrm{mHz}$ ), $D_{L L}$ is given in values per second, $E$ is given in kiloelectronvolt and $\mu$ is given in kiloelectronvolt per nanotesla. These expressions are obtained for quiet and weakly disturbed conditions in the magnetosphere $(\mathrm{Kp} \leq 2)$. During magnetic storms $D_{L L}$ increases, and the expressions obtained here for $D_{L L}$ can change completely.

These results contradict the mechanism of the radial diffusion of particles under the influence of sudden impulses (SI) of the magnetic field and also under the influence of substorm impulses of the electric field, as was suggested by Conwall (1968, 1972).

It is shown that the bulk of the calculations of $D_{L L}$, in the range of $\sim 0.5-1.2 \mathrm{mHz}$ (Pc6), is consistent with spectra of the fluctuations (pulsations) of the electric field at $L \sim 4.5-$ 7.5 during quiet and weakly disturbed periods $(\mathrm{Kp} \leq 2)$ averaged over 7 years for all MLT according to THEMIS data in Liu et al. (2016. These ranges of $f_{\mathrm{d}}$ and $L$ correspond to the trapped protons with energies from $\sim 0.18$ to $\sim 0.7 \mathrm{MeV}$ and electrons with energies from $\sim 0.21$ to $\sim 1.19 \mathrm{MeV}$.

The comparison $D_{L L}$ for protons in a certain region $\{\mu$, $L$ \} with electric field pulsations in the appropriate frequency range shows a close relationship between the radial diffusion of particles and the pulsations of the electric field. For higher frequencies (in the range of Pc5), the experimental spectra of these pulsations are flatter and the spectral density increases by about 1 order of magnitude with the increase in $L$ from
3.5 to 7.5. Therefore, for more energetic particles of the radiation belts corresponding to higher drift frequencies, other dependences of $D_{L L}$ on $\mu$ and $L$ may exist. This applies in particular to the relativistic and ultra-relativistic electrons of the radiation belts.

Because the values of $D_{L L}$ were calculated here only for two short periods (quiet and weakly disturbed) according to only two missions (ISEE-1 and Explorer-45) near the equatorial plane and only for protons in the limited ranges of $\mu$ and $L$, they cannot of course be regarded as complete and final. This work should be continued.

Acknowledgements. The author would like to thank P. Kollmann (Applied Physics Laboratory, Johns Hopkins University) for very important and fruitful comments on and proposals regarding the paper and E. Roussos (Max Planck Institute for Solar System Research) for editing the paper. The author thanks the Kyoto World Data Center for Geomagnetism for providing the Dst indices.

The topical editor, E. Roussos, thanks P. Kollmann, S. Bourdarie, and one anonymous referee for help in evaluating this paper.

\section{References}

Ali, A. F., Elkington, S. R., Tu, W., Ozeke, L. G., Chan, A. A., and Friedel, R. H. W.: Magnetic field power spectra and magnetic radial diffusion coefficients using CRRES magnetometer data, J. Geophys. Res., 120, 973-995, doi:10.1002/2014JA020419, 2015.

Alinejad, N. and Armstrong, T. P.: Radial diffusion of geomagnetically trapped protons observed by the Galileo Energetic Particle Detector, J. Geophys. Res., 111, A09209, doi:10.1029/2005jA011040, 2006.

Berdichevsky, D., Thejappa, G., Fitzenreiter, R. J., Lepping, R. L., Yamamoto, T., Kokubun, S., McEntire, R. W., Williams. D. J., and Lin, R. P.: Widely spaced wave-particle observations during GEOTAIL and Wind magnetic conjunctions in the Earth's ion foreshock with near-radial interplanetary magnetic field, J. Geophys. Res., 104, 463-482, doi:10.1029/1998JA900018, 1999.

Beutier, T., Boscher, D., and France, M.: SALAMMBO: A threedimensional simulation of the proton radiation belt, J. Geophys. Res., 100, 17181-17188, doi:10.1029/94JA02728, 1995.

Blake, J. B., Kolasinski, W. A., Fillius, R. W., and Mullen, E. G.: Injection of electrons and protons with energies of tens of $\mathrm{MeV}$ into $L<3$ on 24 March 1991, Geophys. Res. Lett., 19, 821-824, doi:10.1029/92GL00624, 1992.

Brautigam, D. H. and Albert, J. M.: Radial diffusion analysis of outer radiation belt electrons during the October 9, 1990, magnetic storm, J. Geophys. Res., 105, 291-309, doi:10.1029/1999JA900344, 2000.

Brautigam, D. H., Ginet, G. P., Albert, J. M., Wygant, J. R., Rowland, D. E., Ling, A., and Bass, J.: CRRES electric field power spectra and radial diffusion coefficients, J. Geophys. Res., 110, A02214, doi:10.1029/2004JA010612, 2005.

Chiu, Y. T., Rinaldi, M. A., and Nightingale, R. W.: Toward dynamic modeling of the outer electron radiation belt, J. Geophys. Res., 95, 12069-12074, doi:10.1029/JA095iA08p12069, 1990. 
Claflin, E. S.: Charge-exchange cross sections for hydrogen and helium ions incident on atomic hydrogen: 1 to $1000 \mathrm{keV}$, Rep. TR0059(6260-20)-1, The Aerospace Corporation, El Segundo, CA, USA, 1970.

Cornwall, J. M.: Diffusion processes influenced by conjugate-point wave phenomena, Radio Sci., 3, 740-744, doi:10.1002/rds196837740, 1968.

Cornwall, J. M.: Radial diffusion of ionised helium and protons: A probe for magnetosphere dynamics, J. Geophys. Res., 77, 17561770, doi:10.1029/JA077i010p01756, 1972.

Fälthammar, C.-G.: Effects of time-dependent electric fields on geomagnetically trapped radiation, J. Geophys. Res., 70, 2503-2516, doi:10.1029/JZ070i011p02503, 1965.

Fälthammar, C.-G.: On the transport of trapped particles in the outer magnetosphere, J. Geophys. Res., 71, 1487-1491, doi:10.1029/JZ071i005p01487, 1966.

Fälthammar, C.-G.: Radial diffusion by violation of the third adiabatic invariant, in Earth's Particles and Fields, Ed. B. M. McCormac, Springer, New York, USA, 157-169, 1968.

Fritz, T. A. and Spjeldvik, W. N.: Steady-state observations of geomagnetically trapped energetic heavy ions and their implications for theory, Planet. Space Sci., 29, 1169-1193, doi:10.1016/00320633(81)90123-9, 1981.

Holzworth, R. H. and Mozer, F. S.: Direct evaluation of the radial diffusion coefficient near $L=6$ due to electric field fluctuations, J. Geophys. Res., 84, 2559-2566, doi:10.1029/JA084iA06p02559, 1979.

Jentsch, V.: On the role of external and internal source in generating energy and pitch angle distributions of inner-zone protons, J. Geophys. Res., 86, 701-710, doi:10.1029/JA086iA02p00701, 1981.

Kellogg, P. J.: Van Allen radiation of solar origin, Nature, 183, 1295-1297, doi:10.1038/1831295a0, 1959.

Kepko, L. and Spence, H. E.: Observations of discrete, global magnetospheric oscillations directly driven by solar wind density variations, J. Geophys. Res., 108, 1257, doi:10.1029/2002JA009676, 2003.

Kollmann, P., Roussos, E., Paranicas, C., Krupp, N., Jackman, C. M., Kirsch, E., and Glassmeier K.-H.: Energetic particle phase space densities at Saturn: Cassini observations and interpretations, J. Geophys. Res., 116, A05222, doi:10.1029/2010JA016221, 2011.

Kovtyukh, A. S.: The magnetosphere used as an analyser of spectral form of radiation belt particles, Geomagn. Aeron., 24, 566-570, 1984.

Kovtyukh, A. S.: Interrelation of radial dependence of hardness and shape of proton spectra in the Earth's radiation belts, Geomagn. Aeron., 25, 23-28, 1985a.

Kovtyukh, A. S.: On the form of energy spectrum of protons of the Earth's radiation belts and the mechanisms of its formation, Geomagn. Aeron., 25, 886-892, 1985b.

Kovtyukh, A. S.: Double-peak space-energy structure of the outer ion radiation belt, Geomagn. Aeron., 29, 22-26, 1989.

Kovtyukh, A. S.: The relationship between the pitch-angle and energy distributions of ions in the Earth's radiation belts, Geomagn. Aeron., 33, 453-460, 1994.

Kovtyukh, A. S.: Solar-cycle variations of invariant parameters of ion energy spectra of the Earth's radiation belts, Cosmic Res., 37, 53-64, 1999a.
Kovtyukh, A. S.: Mechanisms of formation of invariant parameters and scaling of ion spectra in a geomagnetic trap, Cosmic Res., 37, 217-229, 1999b.

Kovtyukh, A. S.: Geocorona of hot plasma, Cosmic Res., 39, 527558, doi:10.1023/A:1013074126604, 2001.

Kovtyukh, A. S.: Radial dependence of ionization losses of protons of the Earth's radiation belts, Ann. Geophys., 34, 17-28, doi:10.5194/angeo-34-17-2016, 2016.

Lanzerotti, L. J. and Wolfe, A.: Particle diffusion in the geomagnetosphere: Comparison of estimates from measurements of magnetic and electric field fluctuations, J. Geophys. Res., 85, 23462348, doi:10.1029/JA085iA05p02346, 1980.

Lanzerotti, L. J., Maclennan, C. G., and Schulz, M.: Radial diffusion of outer-zone electrons: An empirical approach to third-invariant violation, J. Geophys. Res., 75, 5351-5371, doi:10.1029/JA075i028p05351, 1970.

Lanzerotti, L. J., Webb, D. C., and Arthur, C. W.: Geomagnetic field fluctuations at synchronous orbit 2. Radial diffusion, J. Geophys. Res., 83, 3866-3870, doi:10.1029/JA083iA08p03866, 1978.

Lindsay, B. G. and Stebbings, R. F.: Charge transfer cross sections for energetic neutral atom data analysis, J. Geophys. Res., 110, A12213, doi:10.1029/2005JA011298, 2005.

Liu, W., Tu, W., Li, X., Sarris, T., Khotyaintsev, Y., Fu, H., Zhang, H., and Shi, Q.: On the calculation of electric diffusion coefficient of radiation belt electrons with in situ electric field measurements by THEMIS, Geophys. Res. Lett., 43, 1023-1030, doi:10.1002/2015GL067398, 2016.

Lyons, L. R. and Williams, D. J.: Quantitative Aspects of Magnetospheric Physics, D. Reidel, Norwell, MA, USA, 1984.

Ma, Q., Li, W., Thorne, R. M., Nishimura, Y., Zhang, X.-J., Reeves, G. D., Kletzing, C. A., Kurth, W. S., Hospodarsky, G. B., Henderson, M. G., Spence, H. E., Baker, D. N., Blake, J. B., Fennell, J. F., and Angelopoulos, V.: Simulation of energy-dependent electron diffusion processes in the Earth's outer radiation belt, J. Geophys. Res., 121, 4217-4231, doi:10.1002/2016JA022507, 2016.

Moldwin, M. B., Downward, L., Rassoul, H. K., Amin, R., and Anderson, R. R.: A new model of the location of the plasmapause: CRRES results, J. Geophys. Res., 107, 1339, doi:10.1029/2001jA009211, 2002.

Nakada, M. P. and Mead, G. D.: Diffusion of protons in the outer radiation belt, J. Geophys. Res., 70, 4777-4791, doi:10.1029/JZ070i019p04777, 1965.

Newkirk, L. L. and Walt, M.: Radial diffusion coefficient for electrons at $1.76<L<5$, J. Geophys. Res., 73, 7231-7236, doi:10.1029/JA073i023p07231, 1968.

Østgaard, N., Mende, S. B., Frey, H. U., Gladstone, G. R., and Lauche, H.: Neutral hydrogen density profiles derived from geocoronal imaging, J. Geophys. Res., 108, 1300, doi:10.1029/2002jA009749, 2003.

Ozeke, L. G., Mann, I. R., Murphy, K. R., Rae, I. J., Milling, D. K., Elkington, S. R., Chan, A. A., and Singer, H. J.: ULF wave derived radiation belt radial diffusion coefficients, J. Geophys. Res., 117, A04222, doi:10.1029/2011JA017463, 2012.

Ozeke, L. G., Mann, I. R., Murphy, K. R., Rae, I. J., and Milling, D. K.: Analytic expressions for ULF wave radiation belt radial diffusion coefficients, J. Geophys. Res., 119, 1587-1605, doi:10.1002/2013JA019204, 2014.

Ozhogin, P., Tu, J., Song, P., and Reinisch, B. W.: Field-aligned distribution of the plasmaspheric electron density: An empirical 
model derived from the IMAGE RPI measurements, J. Geophys. Res., 117, A06225, doi:10.1029/2011jA017330, 2012.

Panasyuk, M. I.: The ion radiation belts: Experiments and models, Effect of Space Weather on Technology Infrastructure, edited by: Daglis, I. A., Kluwer Academic Publ., the Netherlands, 65-90, 2004.

Posch, J. L., Engebretson, M. J., Weatherwax, A. T., Detrick, D. L., Highes, W. J., and Maclennan, C. G.: Characteristics of broadband ULF magnetic pulsations at conjugate cusp latitude stations, J. Geophys. Res., 104, 311-331, doi:10.1029/98JA02722, 1999.

Roederer, J. G.: Dynamics of Geomagnetically Trapped Radiation. Springer, NY, USA, 1970.

Schulz, M. and Lanzerotti, L. J.: Particle Diffusion in the Radiation Belts, Springer, NY, USA, 1974.

Selesnick, R. S., Baker, D. N., Jaynes, A. N., Li, X., Kanekal, S. G., Hudson, M. K., and Kress, B. T.: Inward diffusion and loss of radiation belt protons, J. Geophys. Res., 121, 1969-1978, doi:10.1002/2015JA022154, 2016.

Spjeldvik, W. N.: Equilibrium structure of equatorially mirroring belt protons, J. Geophys. Res., 82, 2801-2808, doi:10.1029/JA082i019p02801, 1977.

Tomassian, A. D., Farley, T. A., and Vampola, A. L.: Inner-zone energetic-electron repopulation by radial diffusion, J. Geophys. Res., 77, 3441-3454, doi:10.1029/JA077i019p03441, 1972.

Tu, W., Elkington, S. R., Li, X., Liu, W., and Bonnell, J.: Quantifying radial diffusion coefficients of radiation belt electrons based on global MHD simulation and spacecraft measurements, J. Geophys. Res., 117, A10210, doi:10.1029/2012JA017901, 2012.

Tverskoy, B. A.: Dynamics of the radiation belts of the Earth, Geomagn. Aeron., 3, 351-368, 1964.
Tverskoy, B. A.: Transport and acceleration of charged particles in the Earth's magnetosphere, Geomagn. Aeron., 5, 517-530, 1965.

Tverskoy, B. A.: Main mechanisms in formation of the Earth's radiation belts, Rev. Geophys., 7, 219-232, doi:10.1029/RG007i001p00219, 1969.

Walt, M.: Introduction to Geomagnetically Trapped Radiation, Cambridge Univ. Press, NY, USA, 1994.

West, H. I., Buck, R. M., and Davidson, G. T.: The dynamics of energetic electrons in the Earth's outer radiation belt during 1968 as observed by the Lawrence Livermore National Laboratory's spectrometer on Ogo 5, J. Geophys. Res., 86, 2111-2142, doi:10.1029/JA086iA04p02111, 1981.

Westphalen, H. and Spjeldvik, W. N.: On the energy dependence of the radial diffusion coefficient and spectra of inner radiation belt particles: Analytic solutions and comparison with numerical results, J. Geophys. Res., 87, 8321-8326, doi:10.1029/JA087iA10p08321, 1982.

Williams, D. J.: Phase space variations of near equatorially mirroring ring current ions, J. Geophys. Res., 86, 189-194, doi:10.1029/JA086iA01p00189, 1981.

Williams, D. J.: Ring current and radiation belts, Rev. Geophys., 25, 570-578, doi:10.1029/RG025i003p00570, 1987.

Williams, D. J. and Frank, L. A.: Intense low-energy ion populations at low equatorial altitudes, J. Geophys. Res., 89, 3903 3911, doi:10.1029/JA089iA06p03903, 1984.

Zoennchen, J. H., Nass, U., and Fahr, H. J.: Exospheric hydrogen density distributions for equinox and summer solstice observed with TWINS1/2 during solar minimum, Ann. Geophys., 31, 513527, doi:10.5194/angeo-31-513-2013, 2013. 